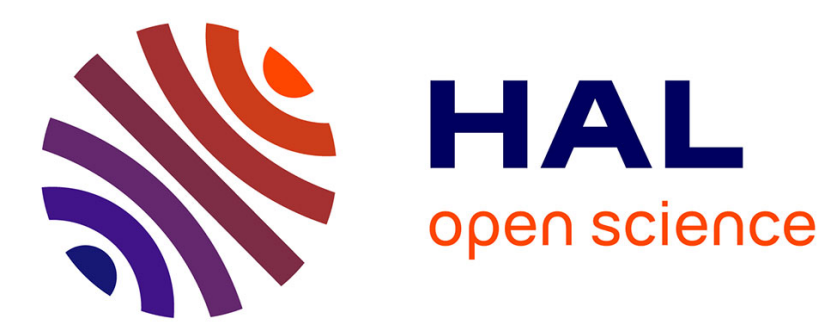

\title{
Les managers raisonnent-ils par options réelles? Une étude exploratoire des déterminants
}

\author{
Marc Fréchet, Hassen Raîs
}

\section{To cite this version:}

Marc Fréchet, Hassen Raîs. Les managers raisonnent-ils par options réelles? Une étude exploratoire des déterminants. XXIV Conférence de l'AIMS, Jun 2015, Paris, France. hal-01764120

\author{
HAL Id: hal-01764120 \\ https://hal.science/hal-01764120
}

Submitted on 11 Apr 2018

HAL is a multi-disciplinary open access archive for the deposit and dissemination of scientific research documents, whether they are published or not. The documents may come from teaching and research institutions in France or abroad, or from public or private research centers.
L'archive ouverte pluridisciplinaire HAL, est destinée au dépôt et à la diffusion de documents scientifiques de niveau recherche, publiés ou non, émanant des établissements d'enseignement et de recherche français ou étrangers, des laboratoires publics ou privés. 


\title{
Les managers raisonnent-ils par options réelles? Une étude exploratoire des déterminants
}

\author{
Fréchet, Marc. Raîs, Hassen \\ CRM. Université Toulouse 1 Capitole \\ marc.frechet@gmail.fr \\ hassen.rais@live.fr
}

\section{Résumé :}

La théorie des options réelles est abondamment évoquée par les travaux en stratégie. Néanmoins, l'impact de cette théorie sur le raisonnement des managers est limité si l'on s'en tient à l'aspect calculatoire de la valorisation des options. Dans le même temps, il apparaît que les décideurs, s'ils n'utilisent pas les options réelles dans leur forme pure, adoptent pourtant des modes de raisonnement qui s'inspirent des grands principes des options réelles. L'objectif de cet article est d'identifier, à partir de quatre grands types d'options, à quels facteurs explicatifs la sensibilité des managers au raisonnement optionnel est rattachée. Par le biais d'une étude empirique, nous montrons que la mobilisation de ces options par les managers dépend étroitement de leur propension à évaluer leurs projets en termes d'alternative et à penser les investissements selon un horizon temporel fini. En revanche, nous mettons en évidence des effets contrastés, selon les catégories d'options, de l'incertitude ressentie, de la pression à l'innovation, et de la veille environnementale menée au sein de l'entreprise.

Mots-clés : option réelle, flexibilité, raisonnement optionnel, décision. 


\section{Les managers raisonnent-ils par options réelles ? Une étude exploratoire des déterminants}

\section{INTRODUCTION}

Les options réelles ont reçu une attention soutenue dans la recherche académique. Issues de la finance, elles ont fait l'objet d'amples développement dans le champ de la stratégie (Durand et al., 2002), qu'il s'agisse, entre autres, des projets de R\&D (Wouters et al., 2011), des alliances stratégiques (Kogut, 1991 ; McCarther et al., 2011), ou de la manière dont les organisations configurent un portefeuille d'options (Barnett, 2008). Sous cet angle, la stratégie semble être irriguée par l'un des développements majeurs de la théorie financière. Plus encore, selon Myers (1996), la théorie des options constituerait l'outil idéal pour faire dialoguer les deux disciplines.

Pourtant, l'apparente diffusion de la notion d'options contraste fortement avec ses apports effectifs dans le champ de l'aide à la décision. En réalité, l'utilisation des options réelles dans le monde de l'entreprise reste très limitée, et les enquêtes empiriques font état d'une faible diffusion de ces outils chez les décideurs (Bowman et Moskowitz, 2001. Triantis, 2005). Pour l'expliquer, deux grandes difficultés de diffusion et de mise en œuvre des options réelles dans l'entreprise ont été avancées. D'une part, les modèles actuels de valorisation d'options réelles seraient perçus comme trop complexes par les managers. D'autre part, les difficultés d'adaptation des modèles d'options financières aux décisions concrètes d'investissement limiteraient leur intérêt intrinsèque (Myers, 1996).

Face à ces difficultés, les chercheurs ont progressivement préconisé une utilisation plus qualitative des options réelles (Krychowski, 2007). Ils ont observé que, si les entreprises sont réticentes à utiliser les modèles de valorisation d'option, un certain nombre d'entre elles ont intégré la logique optionnelle dans leur manière d'appréhender et structurer les projets d'investissement (Triantis, 2005). C'est-à-dire que si l'utilisation des modèles quantitatifs de valorisation d'option est hors de portée de la plupart des managers, les options réelles sont néanmoins porteuses d'un type de raisonnement, d'une démarche, voire d'un état d'esprit que 
les décideurs peuvent s'approprier. L'utilisation d'un raisonnement intégrant les grands principes des options réelles a dès lors fait l'objet d'une attention naissante de la part des chercheurs sous les termes de «Real Option Reasoning » (ROR) (McGrath, 1999) ou « Real Option Thinking » (Franqueira, Houmb, Daneva, 2010).

Il reste que le contenu de ce peut être le ROR, ses dimensions-clés, ou encore ses déterminant demeure méconnu. Les études empiriques sur la question, rares, se concentrent sur l'intérêt que peut revêtir le ROR et ne tentent généralement pas de mesurer la propension des managers à l'adopter. Dans ce paysage, les travaux se concentrant sur les causes ou l'impact de l'adoption d'un raisonnement optionnel demeurent l'exception. Notre étude vise à combler cette lacune, en abondant dans le sens des études reconnaissant l'importance d'un raisonnement optionnel, indépendamment des conditions de formalisation mathématique que lui avait conférée la théorie financière.

Par le biais d'une enquête par questionnaire, nous mesurons quatre grandes catégories d'options telles que les décideurs déclarent les mobiliser dans leur réflexion (options de modifications, de report, de croissance et de composition ; Trigorgis, 1996). Conformément à la théorie, nous montrons que la mobilisation de ces options par les managers dépend étroitement de leur propension à évaluer leurs projets en termes d'alternative et à penser les investissements selon un horizon temporel fini. En revanche, nous mettons en évidence des effets contrastés, selon les catégories d'option, de l'incertitude ressentie, de la pression à l'innovation, et de la veille environnementale menée au sein de l'entreprise.

La suite de l'article est organisée de la manière suivante. Une partie théorique vise à rappeler les principes d'utilisation des options ainsi que le glissement que se sont autorisés les chercheurs vers la notion de raisonnement optionnel. Une deuxième partie est consacrée à la formulation des hypothèses sur les déterminants du raisonnement optionnel. Une troisième partie est dès lors dédiée à la mise en place du protocole empirique et au test des hypothèses. Une quatrième partie est, par suite, employée à la discussion des résultats. Enfin, la conclusion, revient sur les apports, les limites et les voies de recherches ultérieures. 


\section{OPTIONS REELLES ET "REAL OPTION REASONING"}

\subsection{LA NOTION D'OPTION REELLE}

Les options réelles sont nées des insuffisances des méthodes classiques d'évaluation des investissements. Le principal inconvénient de ces méthodes réside dans la vision déterministe et linéaire qu'elles promeuvent (Savage, 2009). Un seul déroulement est prévu, il n'y a pas de bifurcation intermédiaire. Le projet est alors censé être retenu si la valeur actuelle nette est positive. Au contraire, en adoptant un schéma arborescent, la méthode d'Evaluation par les Options Réelles fait émerger différents scénarii et permet d'identifier les jalons du projet; elle s'adapte parfaitement aux projets d'investissements s'effectuant par étapes (Brealey et Myers, 2010). Dès lors que le financement se fait par étapes, il existe une possibilité d'adapter le projet aux conditions futures ce qui constitue une «flexibilité » dont il faut tenir compte lors de l'évaluation initiale.

Il existe par conséquent une flexibilité managériale, c'est-à-dire une capacité du manager à adapter la décision d'investissement par un ajustement aux conditions existantes de marché, comme par la modification des hypothèses initiales de simulation du projet et/ou de ces objectifs. Cette possibilité, cette flexibilité - en un mot cette option - présente un intérêt qui lui confère une valeur qu'un manager pourra ou non accepter d'acquitter pour la détenir (Driouch et al. 2012).

L'option réelle, par analogie avec l'option financière, confère le droit, et non l'obligation, de prendre une décision stratégique d'investissement relative à un actif sousjacent non financier. Ce sous-jacent peut être un projet ou un actif réel tel qu'un bien d'équipement, une usine de production, projet $R \& D$, une activité en phase de démarrage ou de croissance, ou bien encore un droit de propriété intellectuelle (Hurry, Miller et Bowman, 1992 ; Kim et Kogut, 1991). De ce point de vue, les grands instruments stratégiques, dès lors qu'ils confèrent une possibilité de reporter, modifier ou décomposer un choix, sont porteurs d'options réelles.

Sur un plan opérationnel, les options peuvent prendre différentes formes, c'est-à-dire consister en certaines possibilités de flexibilité relativement aux choix stratégiques. La littérature en propose différents types que nous avons regroupés en quatre catégories (Trigeorgis, 1996) :

- Les options de modification : il s'agit du droit de modifier un projet, en y ajoutant ou 
en y soustrayant des ressources et des possibilités. Comprises au sens large, ces options comprennent le redimensionnement d'un projet mais aussi la possibilité pure et simple de l'abandonner;

- Les options de report : il s'agit du droit de reporter le point de départ d'un projet. Ce type d'option présente une valeur en ce qu'elle permet de diminuer l'incertitude inhérente au choix d'investissement ;

- Les options de croissance : il s'agit de la possibilité d'utiliser un investissement pour ouvrir de nouvelles possibilités de développement. L'investissement dans l'option s'apparente à un droit de rester en jeu pour savoir si le projet mérite d'être mené plus avant ;

- Les options composées : elles existent lorsque le projet est décomposé en plusieurs étapes ponctuées de jalons et dont l'issue dépend de la réussite des étapes précédentes. Ces options sont surtout utilisées en R\&D pour les choix d'investissements dans de nouveaux prototypes ou de nouvelles technologies, ainsi que dans l'industrie pharmaceutique pour les phases de tests de médicaments.

\subsection{DIFFICULTES OPERATIONNELLES DES OPTIONS REELLES}

Une première difficulté est que les options réelles sont difficiles à identifier car, contrairement aux options financières, elles ne sont pas formalisées par un contrat (Myers, 1996; Copeland \&Keenan, 1998). Dans la pratique, l'identification des options réelles est malaisée, car les dirigeants sont peu familiers avec la logique optionnelle. Kemna (1993) explique ainsi que l'on observe dans le monde de l'entreprise une confusion entre les alternatives, et les options contenues dans ces alternatives : il y a souvent des malentendus, car les managers considèrent souvent que les alternatives constituent des options. Même pour les managers ayant été sensibilisé au raisonnement optionnel, la complexité de la situation est telle qu'il n'est pas toujours facile de reconnaître les options réelles.

Une seconde difficulté des options réelles concerne leur valorisation. La nécessité de valoriser l'option provient du fait que la flexibilité est rarement gratuite. Tout l'intérêt des options réelles est alors de valoriser cette flexibilité, et de pouvoir la comparer à leur coût. Lorsque les applications concrètes des options réelles sont abordées, il apparaît que la valorisation de l'option est bien souvent nécessaire à la prise de décision. C'est par exemple le cas lorsque l'existence d'une option de croissance peut justifier - ou non - le lancement d'un 
projet innovant. De même, l'option d'élargir le périmètre d'un projet ou l'option de modifier l'usage d'un projet sont appréciables, mais nécessitent au départ des coûts d'investissement ou des temps de développement supplémentaires.

Compte tenu de ces difficultés, les chercheurs suggèrent de contourner le problème de l'identification et de la valorisation en affirmant que les options réelles peuvent être utilisées comme un cadre conceptuel et comme mode de pensée, plus que comme un outil de valorisation (Sharp, 1991; Miller \& Waller, 2003; Fichman et al., 2005).

\subsection{LE RAISONNEMENT PAR LES OPTIONS REELLES (ROR)}

Le raisonnement par les options réelles s'entend comme le fait, par les décideurs, d'utiliser les options réelles comme cadre d'analyse et comme outil de réflexion sans pour autant se livrer à une rigoureuse formalisation, ni même à des calculs détaillés. Il suffit que les décideurs aient conscience des grandes caractéristiques des options possibles pour voir s’ils souhaitent se constituer un portefeuille d'options et s'ils souhaitent y investir.

En pratique, le développement de la méthode en temps continu et le recours aux arbres de décision (Copeland et Antikarov, 2001 et 2003) répondent à cette logique. Très généralement, une représentation arborescente des projets dont l'appropriation est aisée pour tous les participants est révélatrice d'un raisonnement optionnel (McGrath, 1999). Par référence aux quatre catégories d'options évoquées plus haut (modification, report, croissance, décomposition), le fait qu'un manager envisage, par exemple, un investissement comme le moyen de déverrouiller des possibilités sur lesquelles il conviendra de se prononcer le moment venu est typique d'un raisonnement optionnel sans qu'une évaluation chiffrée n'intervienne nécessairement. Le raisonnement par options réelles (ROR) procure donc une image plus étendue du projet en capturant la capacité des managers à modifier ou optimiser les activités et opérations au fur et à mesure que l'information devient disponible ou que les incertitudes sont levées. Ainsi, l'analyse par les options réelles, par le nouveau raisonnement qu'elle encourage, peut durablement modifier le processus de pensée à l'intérieur des organisations.

\section{HYPOTHESES DE RECHERCHE}

L'idée directrice de notre travail est donc que les options réelles peuvent être utilisées par les décideurs comme un cadre conceptuel, qui permettra de structurer la décision 
d'investissement, et d'imposer plus de discipline dans le pilotage du projet (Bellalah, 2001). Cette utilisation est d'ailleurs possible dans une très grande diversité de décisions d'investissement (Triantis, Borison, 2001). Le type raisonnement mené dépendra du niveau d'analyse retenu, de l'horizon de temps envisagé, et de la nature du projet. Il n'existe donc pas d'analyse optionnelle standard. Cependant le raisonnement par options réelles suppose que de nombreux investissements peuvent être effectués par étapes, en conservant une souplesse d'adaptation aux conditions futures. Cette flexibilité est porteuse de valeur car elle augmente les chances de succès et limite les risques de pertes des projets d'investissement (Bowman, Hurry, 1993). Sous ces conditions, on peut dès lors estimer que la sensibilité des décideurs au raisonnement optionnel est dépendante d'un certain nombre de variables liées à la nature même du raisonnement, mais aussi aux habitudes de réflexion des managers face aux projets d'investissement. Ces différents facteurs sont abordés dans les lignes qui suivent. Les trois premiers sont des facteurs classiques (Krychowski, 2007) présentés dans les recherches sur les options réelles. Les deux autres sont des facteurs que l'on propose et que l'on teste dans leur capacité à expliquer le ROR.

\subsection{LES ALTERNATIVES}

Un raisonnement en termes d'alternatives est essentiel en matière de raisonnement par options réelles (Kemna, 1993 ; Brealey et Myers, 2010). Pour que l'option prenne de la valeur, il faut que d'autres décisions soient possibles, ce qui permet d'inscrire la stratégie optionnelle dans une théorie des choix rationnels. C'est ce qui donne en effet du sens au choix de poursuivre une stratégie ou de l'arrêter. Par exemple, il est difficile d'examiner l'intérêt d'une option technologique si on ne définit pas quels seraient les gains consécutifs à d'autres options ou d'autres stratégies. Le choix rationnel s'évalue toujours en fonction des coûts d'opportunité. En conséquence, il est essentiel de comprendre les représentations du décideur pour repérer par rapport à quelle(s) alternative(s) il évalue le bien fondé de sa stratégie d'option, d'où la première hypothèse.

Selon cette logique, les décideurs qui n'ont pas l'habitude de raisonner en termes d'alternatives sur leurs projets adopteront probablement moins un raisonnement de type optionnel. La vision classique des investissements les incite à rechercher un résultat sans qu'ils doivent s'interroger continûment sur les possibilités parallèles qui peuvent surgir ou disparaître. On posera donc que la propension des managers à raisonner en termes 
d'alternatives sur leurs projets d'investissement est étroitement associée à l'adoption du ROR. C'est-à-dire qu'en cas d'alternative, les managers seront davantage sensibles à la présence d'options formulées comme telles. Comme il existe différents types d'options (quatre dans notre cas), il est cependant difficile de soutenir ex ante que l'utilisation d'alternatives agit sur toutes les options. Nous formulons donc une hypothèse à deux niveaux, l'un fort, selon lequel toutes les options sont affectées, et l'autre faible, selon lequel au moins l'une des options est influencée.

H1forte : L'évaluation des projets en termes d'alternatives est positivement associée à l'ensemble des options perçues par les décideurs.

H1faible: L'évaluation des projets en termes d'alternatives influence positivement un ou plusieurs aspects du raisonnement optionnel par les décideurs.

\subsection{L'HORIZON TEMPOREL}

Classiquement, on estime qu'une décision stratégique n'est optionnelle que dans la mesure où le décideur limite son espace temporel de manière à pouvoir comparer un choix avec un autre (Durand et al., 2002). En d'autres termes, il faut que le futur soit borné. Sans cette hypothèse, il est impossible de déterminer en quoi un choix peut être meilleur qu'un autre, et donc en quoi une option possède une valeur (Cohendet, Llerena, 1999). La prise de décision concrète par les managers doit, pensons-nous, faire écho à cette condition qui existe dans la théorie formalisée des options réelles. Même si le rôle de l'horizon temporel peut être atténué, un décideur ne s'orientera pas avec la même force vers un raisonnement optionnel s'il ne spécifie pas explicitement un horizon temporel fini. Par exemple, en présence d'un investissement de recherche, il pourra raisonner sur une période stratégique donnée (en général 5 à 10 ans). Dans ce cas, il évaluera les opportunités que pourrait révéler ce projet ou réfléchira aux possibilités de l'abandonner si des résultats ne sont pas obtenus. Cependant, s’il ne raisonne pas selon un horizon temporel défini, sa démarche sera plus difficile. En effet, il sera toujours possible de pouvoir espérer que n'importe quelle recherche puisse avoir un jour une certaine valeur, et qu'il est par conséquent inutile de mobiliser la notion d'option (Folta, Miller, 1999). De manière similaire, les opportunités ouvertes par le projet seront rendues bien moins fortes car, avec le temps, la concurrence pourra générer les possibilités avant 
l'entreprise qui investit dans le projet. Par conséquent, la fixation d'un horizon temporel fini est un stimulant à la réflexion en termes d'options.

H2forte : La réflexion en termes d'horizon temporel fini sur les projets est positivement associée à l'ensemble des options perçues par les décideurs.

H2faible : La réflexion en termes d'horizon temporel fini sur les projets influence positivement une ou plusieurs aspects du raisonnement optionnel par les décideurs.

\subsection{L'INCERTITUDE}

L'incertitude informationnelle est traditionnellement rattachée à la constitution d'options. Cette incertitude existe dès lors que l'avenir est probabilisable au sens où les acteurs savent que quelque chose peut se réaliser mais pas avec quel degré de probabilité (Harchaoui, Lasserre, 2001). C'est à raison de cette incertitude que le raisonnement optionnel montre son intérêt. Si aucune incertitude n'existe, les décideurs n'ont qu'une faible incitation à rechercher une flexibilité dans leurs décisions d'investissement, hormis le besoin de corriger d'éventuelles erreurs. En revanche, lorsque l'environnement est perçu comme changeant, le besoin d'adaptation des décisions, et donc l'utilisation des options, trouve un intérêt pour les managers (Li et Chi, 2013). On peut donc supposer qu'ils seront davantage enclins à rechercher une forme de flexibilité dans leurs décisions.

H3forte : L'incertitude perçue de l'environnement est positivement associée à l'ensemble des options perçues par les décideurs.

H3faible : L'incertitude perçue de l'environnement influence positivement un ou plusieurs aspects du raisonnement optionnel par les décideurs.

\subsection{LA PRESSION A L'INNOVATION}

La flexibilité est souvent considérée comme une caractéristique fondamentale des organisations innovantes. Non seulement l'innovation requiert de la flexibilité mais, en outre, la flexibilité de l'organisation est souvent le fruit de l'innovation. L'apport principal du ROR réside dans le fait qu'il reconnaît d'emblée que l'entreprise pourra ajuster sa stratégie d'investissement à l'innovation (Krychowski, 2008). Symétriquement, le caractère incertain 
des processus innovants font de l'innovation un domaine qui se prête particulièrement bien à une approche optionnelle (Perez, Berard, 2009). Ainsi, la mobilisation des options réelles dépend de la pression à l'innovation d'un secteur particulier. Les options réelles sont considérées comme particulièrement adaptées lorsque l'entreprise s'oriente vers des stratégies d'innovation. Le besoin intrinsèque de flexibilité des projets innovants rend attractif la possibilité de différer certains choix ou de les modifier. Pour cette raison, on peut supposer qu'une plus grande pression à l'innovation va engager les managers vers un type de raisonnement davantage optionnel.

H4forte : La pression à l'innovation est positivement associée à l'ensemble des options perçues par les décideurs.

H4faible : La pression à l'innovation influence positivement un ou plusieurs aspects du raisonnement optionnel par les décideurs.

\subsection{LA VEILLE ENVIRONNEMENTALE}

Enfin, la veille environnementale est également un facteur susceptible d'influencer le choix des options. En effet, la réceptivité des managers aux signaux extérieurs est un élément reconnu comme de nature à favoriser le raisonnement optionnel (Williams, 1991). Les managers qui établissent une veille quant aux signaux extérieurs sont davantage conscients des besoins de flexibilité en matière de choix stratégiques. Ils peuvent donc raisonnablement être supposés plus sensibles à la possibilité de reporter, modifier ou décomposer un projet. Par exemple, la mise en place d'une démarche optionnelle dans un projet de R\&D implique que l'organisation soit en mesure, d'une part, d'identifier les sources d'incertitude et de recueillir l'information et, d'autre part, d'identifier les risques et les opportunités associés au projet. Cette règle semble aller de soi ; pourtant la connaissance de son environnement par une organisation n'est pas évidente. Pour cette raison, et dans le cadre d'une démarche optionnelle, la veille stratégique et concurrentielle est un outil primordial (Perez, Berard, 2009).

H5forte : La veille environnementale est positivement associée à l'ensemble des options perçues par les décideurs. 
H5faible: La veille environnementale influence positivement un ou plusieurs aspects du raisonnement optionnel par les décideurs.

\section{METHODE}

\subsection{DONNEES}

Cette recherche s'est faite sur la base d'une enquête par questionnaire administrée auprès d'entreprises de Midi-Pyrénées. L'échantillon d'entreprises a été extrait, selon une double stratification par taille et par secteur d'activité, à partir de la base de données Point Risk qui recense et présente la structure et les données financières de plus de 1,2 millions d'entreprises Françaises.

Le questionnaire adressé au dirigeant recueille les informations relatives à la posture liée au raisonnement par options réelles adopté et développé lors de la gestion des projets. Les entreprises ont été approchées par email et téléphone. Lorsqu'elles ne répondaient pas, les entreprises étaient relancées jusqu'à deux fois par les mêmes moyens. La collecte s'est déroulée sur plus un trimestre, de septembre à décembre 2014.

Un total de 360 questionnaires exploitables a été recueilli. Afin d'estimer un éventuel biais de non réponse, nous avons comparé les répondants précoces de ceux qui avaient répondu tardivement. Des tests de moyenne n'ont fait ressortir aucune différence significative suggérant que, du point de vue de cette vérification, il n'existait pas de biais décelable.

\subsection{ARIABLES}

L'incertitude perçue a été mesurée par trois items mesurés selon une échelle de likert à 7 points («Il est difficile de prédire les évolutions de l'environnement»; «Les préférences des clients sont changeantes »; «Dans l'ensemble, notre environnement est incertain »). Les analyses factorielles font ressortir une structure unidimensionnelle ainsi qu'une fiabilité acceptable dans le cadre d'une étude exploratoire (alpha de Cronbach =0,66).

La veille environnementale a été mesurée par trois items sur une échelle de likert à 7 points («Nous sommes attentifs aux changements environnementaux (société, législation, etc.) », « Nous sommes vigilants quant à l'émergence de nouvelles opportunités », « Nous sommes attentifs aux changements du marché, y compris dans les industries qui ne sont pas 
les nôtres »). L'échelle est unifactorielle et présente une consistance acceptable (alpha de Cronbach $=0,70)$.

La pression à l'innovation a été appréhendée au travers de trois items selon une échelle de likert à 7 points («Direz-vous que, dans votre secteur d'activité...», «... l'innovation est un élément de succès », « ... la réussite repose sur le lancement de nouveaux produits », « ... la recherche de nouveaux marchés est essentielle »). La structure de l'échelle est unifactorielle et fait ressortir une fiabilité correcte (alpha de Cronbach $=0,75$ ).

La variable d'horizon temporel est mesurée par trois items selon une échelle de likert à 7 points (« Pour prendre une grande décision... », « ... je veille à ce que l'évaluation du projet intègre une date butoir », « ... je m'efforce de fixer un horizon temporel déterminé pour guider ma réflexion », «...tout projet s'évalue pour moi sur une période déterminée »). L'échelle est unifactorielle et sa fiabilité apparaît comme bonne (alpha de Cronbach =0,81).

La variable de raisonnement en termes d'alternatives est appréhendée par trois items sur une échelle de likert à 7 points («Pour prendre une grande décision...», «... j'envisage le maximum d'alternatives », « ... je reste attentif à l'éventail le plus large possible de solutions », « ... j'essaye de comparer les différentes options »). L'échelle est unifactorielle et sa fiabilité satisfaisante (alpha de Cronbach $=0,83$ ).

\section{Variables dépendantes}

La mobilisation des options a fait l'objet d'une série de questions qui été introduite par la question générale suivante : «Dans certains cas, une grande décision offre néanmoins la possibilité de conserver une marge de manœuvre en ce que tout n'a pas à être décidé immédiatement. Il en résulte une possibilité de choisir plus tard. Comment percevez-vous ce cas de figure ?».

Les options de croissance ont été mesurées par le biais de trois items sur une échelle de likert à 7 points («Pour moi il est important de voir qu'un projet d'investissement peut ...», « ...offrir la possibilité de déverrouiller d'autres projets futurs », « ... être la première chaine d'une série de projets interdépendants »). L'échelle est bien unifactorielle mais la fiabilité semble moyenne, étant toutefois rappelé la dimension exploratoire de l'étude (alpha de Cronbach $=0,68)$.

Les options de modification des projets ont été mesurées par trois items sur une échelle de likert à 7 points ( «J'attache une importance particulière à cette flexibilité pour ce 
qui est d'éventuellement ...», « ... modifier la nature des projets », « ... décider d'abandonner des projets », «...changer l'échelle des projets (investissements supplémentaires ou moindres) »). L'analyse factorielle fait ressortir une structure unidmensionnelle la fiabilité est acceptable dans le cadre d'une étude exploratoire (alpha de Crombach $=0,67$ ).

Les options de retardement du projet ont été mesurées par trois items sur une échelle de likert à 7 points («Je suis habituellement sensible à la possibilité de repousser la réalisation d'un projet dès lors que ... », « ... les incertitudes technologiques seront réduites », «... les risques d'échec du projet proprement dit seront diminués », « ... les prérequis du projet seront davantage clairs »). L'échelle est unidimensionnelle et sa fiabilité est bonne (alpha de Cronbach $=0,80$ ).

Les options de décomposition ont été mesurées par trois items sur une échelle de likert à 7 points («Face à une décision d'investissement dans un projet, j'essaie de voir dans quelle mesure ce projet ...», «... pourrait être financé par étapes successives », «... pourrait faire l'objet d'un financement par pallier», « ... pourrait être décomposé en série de plus petits projets »). La structure est unidmensionnelleet la consistance interne bonnne (alpha de Cronbach $=0,74)$.

\subsection{STATISTIQUES DESCRIPTIVES}

Le tableau 1 présente les données descriptives tandis que le tableau 2 présente les corrélations. Les données descriptives sont difficilement lisibles dans la mesure où les variables sont essentiellement issues de scores factoriels. Le tableau de corrélations fait ressortir des liens que l'on pourrait qualifier de plutôt faibles, et qu'en tout état de cause, aucun problème de colinéarité n'est à suspecter. 
Tableau 1 - Statistiques descriptives

\begin{tabular}{lcccc}
\hline Variable & Moy. & Std. err. & Min. & Max. \\
\hline Modif & 0 & 1.322 & -3.250 & 2.643 \\
Report & 0 & 1.503 & -3.859 & 2.454 \\
Croiss & 0 & 1.292 & -2.984 & 2.296 \\
Decompo & 0 & 1.369 & -3.446 & 2.464 \\
incert & 0 & 1.363 & -3.965 & 2.643 \\
prestechno & 0 & 1.169 & -3.234 & 2.034 \\
Altern & 0 & 1.384 & -3.902 & 2.247 \\
Temps & 0 & 1.034 & -2.984 & 1.948 \\
Envir & 0 & 1.237 & -3.260 & 2.367 \\
LNemp & 1,696 & 1.148 & 0 & 5.192 \\
LNca & 7,629 & 1.760 & .454 & 11.346 \\
Age & 45 & 10.222 & 21 & 65 \\
Etudes & 3 & 2.171 & 0 & 8 \\
\hline
\end{tabular}




\begin{tabular}{|c|c|c|c|c|c|c|c|c|c|c|c|c|}
\hline & modif & report & croiss & decompo & incert & prestechno & altern & temporel & envir & LNemp & LNca & $\bar{a}$ \\
\hline \multicolumn{13}{|l|}{ modif } \\
\hline report & $0.2557 * * *$ & & & & & & & & & & & \\
\hline croiss & $0.2564 * * *$ & $0.2039 * *$ & & & & & & & & & & \\
\hline decompo & $0.2275 * * *$ & $0.1479 * *$ & $0.2969 * * *$ & & & & & & & & & \\
\hline incert & $0.1095^{*}$ & $0.1372 * *$ & $0.1771 * * *$ & $0.0968^{*}$ & & & & & & & & \\
\hline prestechno & $0.1383 * *$ & $0.1149 *$ & $0.3847 * * *$ & $0.2014 * * *$ & $0.1637 * * *$ & & & & & & & \\
\hline altern & $0.2841 * * *$ & $0.2263 * *$ & $0.3595 * * *$ & $0.2521 * * *$ & $0.1087^{*}$ & $0.2161 * * *$ & & & & & & \\
\hline temporel & $0.1777 * *$ & $0.2336 * *$ & $0.2170 * *$ & $0.1655^{* *}$ & $0.1347 * *$ & $0.1096^{*}$ & $0.2674 * * *$ & & & & & \\
\hline envir & $0.1001 *$ & $0.1505^{* *}$ & $0.3342 * * *$ & $0.1306^{* *}$ & $0.1476^{* *}$ & $0.2656 * * *$ & $0.3656 * * *$ & $0.1734 * *$ & & & & \\
\hline LNemp & 0.0387 & -0.0082 & 0.0093 & -0.0524 & -0.0137 & 0.0008 & 0.0747 & 0.0837 & $0.1058 *$ & & & \\
\hline LNca & -0.0501 & -0.0371 & 0.0213 & -0.0868 & -0.0611 & -0.0386 & 0.0783 & 0.0176 & -0.0343 & $0.3193 * * *$ & & \\
\hline age & 0.0737 & 0.0125 & -0.0433 & 0.0171 & -0.0308 & -0.0108 & 0.0300 & 0.0711 & 0.0219 & $0.1152 *$ & $0.1362 * *$ & \\
\hline \multirow[t]{2}{*}{ etudes } & $-0.0943 *$ & -0.0988 & -0.0953 & -0.0555 & -0.0555 & -0.0415 & 0.0257 & - & -0.0676 & 0.0632 & 0.0421 & - \\
\hline & & & & & & & & $0.1887 * *$ & & & & $0 .($ \\
\hline
\end{tabular}




\subsection{Modeles et Resultats}

Afin de tester les hypothèses, quatre modèles ont été élaborés. Ils sont présentés dans le tableau 3. Chacun comprend, comme variable dépendante, l'une des quatre options identifiées. Le modèle 1 vise à expliquer la mobilisation des options de modification. Le modèle 2 est dédié aux options de report. Le modèle 3 concerne les options de croissance tandis que le modèle 4 explique les options de décomposition. Chacun de ces modèles permet de tester les hypothèses dans leur forme atténuée tandis que le test cumulatif sur l'ensemble des modèles permet de tester les hypothèses dans leur formulation forte.

Tableau 3 - Régressions linéaires sur les différents types d'options

\begin{tabular}{lcccc}
\hline \multirow{2}{*}{ Variables } & Modèle 1 & Modèle 2 & Modèle 3 & Modèle 4 \\
\cline { 2 - 5 } incert & Modif & report & croiss & decompo \\
\hline prestechno & $.012(.054)$ & $.128(.058)^{*}$ & $.087(.052)$ & $.046(.053)$ \\
altern & $.267(.053)^{* * *}$ & $.154(.058)^{* *}$ & $.171(.052)^{* * *}$ & $.144(.054)^{* *}$ \\
temporel & $.120(.068)^{* *}$ & $.161(.074)^{* * *}$ & $.067(.067)^{*}$ & $.090(.067) \dagger$ \\
envir & $-.086(.059)$ & $.067(.064)$ & $.193(.058)^{* * *}$ & $-.044(.059)$ \\
LNemp & $.000(.066)$ & $-.122(.071)^{*}$ & $-.044(.064)$ & $-.089(.066)$ \\
LNca & $-.066(.044)$ & $.023(.045)$ & $.041(.041)$ & $-.082(.521)$ \\
age & $.067(.008)$ & $.014(.009)$ & $-.064(.007)$ & $-.003(.008)$ \\
etudes & $-.056(.037)$ & $-.113(.042)$ & $-.045(.038)$ & $.066(.038)$ \\
Const. & $.429(.473)$ & $.243(.514)$ & $-.135(.303)$ & $.698(.429)$ \\
& & & & \\
\hline F & & & & \\
R2 & $5,43 * * *$ & $6,33 * * *$ & $21,03 * * *$ & $5,02 * * *$ \\
\hline$* p<0,05$ & $12,26 \%$ & $14,07 \%$ & $28,57 \%$ & $9,96 \%$ \\
$* * * 0,01$ & & & & \\
$* * * 0,001$ & & & & \\
\hline
\end{tabular}

Il ressort avec suffisamment de netteté que l'habitude de raisonner, sur les projets d'investissement, en termes d'alternative, a une influence sur l'utilisation de tous les types 
d'options étudiés. Il s'en déduit que l'hypothèse H1 est validée dans sa version forte. Des résultats presque identiques apparaissent avec un raisonnement en termes d'horizon temporel fini. Trois des quatre options sont nettement dépendantes de cette démarche. En revanche, les résultats sont moins nets pour les options de décomposition. Dans ce cas, l'influence de l'utilisation d'un horizon temporel fini n'influence que modérément l'utilisation de l'option $(\beta=.090, p=0,71)$. Toutefois, cet effet limité doit être rapproché de la variance totale expliquée par le modèle, qui est la plus basse de tous les modèles testés. L'étude est par ailleurs profondément exploratoire. Par prudence, il nous semble plus judicieux de ne pas rejeter l'hypothèse H0. Sous la réserve précédemment énoncée, nous proposons donc d'admettre un effet global de l'horizon temporel et de valider $\mathrm{H} 2$, là encore dans sa version forte.

En revanche, les résultats des régressions montrent que l'incertitude n'a qu'une influence limitée sur un seul des types d'option, celles de croissance $(\beta=.128, p<0,05)$. De fait, l'hypothèse $\mathrm{H} 3$ est fermement rejetée dans sa version forte et à peine validée dans sa version faible. Enfin, concernant les deux dernières hypothèses relatives à la pression à l'innovation et à la veille environnementale qui sont deux variables que l'on a rajouté aux variables traditionnelles et que l'on souhaite tester, on remarque que, la pression à l'innovation a une influence nette sur les options de croissance et de décomposition (respectivement $\beta=.271, p<0,001 ; \beta=.167, p<0,001$ ) sans en avoir sur les autres options. Son influence étant établie de manière limitée, on est conduit à valider l'hypothèse H4 dans sa forme faible. On relève que la veille environnementale n'influence que les options de croissance, bien que ce soit de manière marquée $(\beta=.193, p<0,001)$. Nous validons par conséquent l'hypothèse $\mathrm{H} 5$ dans sa forme faible tout en la rejetant dans sa formulation forte.

\section{DISCUSSION}

Si les travaux académiques invoquent depuis longtemps l'intérêt des options réelles dans la réflexion stratégique, la manière que peuvent avoir les managers de s'approprier le raisonnement optionnel demeure méconnue (Philippe, 2004). L'objectif de cette étude était de contribuer à l'étude émergente du «real option reasoning» par les managers. Au travers d'une enquête menée auprès de dirigeants d'entreprises françaises, nous montrons que le raisonnement optionnel est étroitement dépendant de la conception des projets d'investissement en termes d'alternatives et d'horizon temporel fini. Touts les types d'options apparaissent sensibles à ces déterminants. En revanche, des facteurs comme l'incertitude perçue, la vigilance à l'environnement ou encore la pression à l'innovation n'ont pas des 
effets homogènes et varient selon les catégories d'option. A partir de ces résultats, on peut avancer que la présente étude contribue à la littérature sur trois points.

Premièrement, l'étude confirme la réalité d'un « real option reasoning » chez les managers (Blum, 2012), qui se conçoit comme la recherche d'une flexibilité stratégique dans le développement des projets. Le raisonnement par option réelle s'exprime sous la forme d'un intérêt porté à la possibilité de modifier, reporter, décomposer un projet, ou y investir avec l'idée que de nouvelles opportunités, non encore aperçues, vont en émerger au fil du temps. Cette manière de concevoir les investissements est étroitement dépendante d'une conception des projets en termes d'alternatives. Dit autrement, le raisonnement par options réelles sera d'autant plus présent que les managers ont tendance à penser leurs projets sous forme d'arborescences, de bifurcations, ou même de possibilités parallèles. Dans une moindre mesure, et conformément à la théorie des options réelles (Adner, Levinthal, 2004), les managers sont d'autant plus poussés vers le raisonnement par options réelles qu'ils s'imposent des horizons temporels finis pour évaluer leurs projets d'investissement.

Deuxièmement, notre étude semble en revanche marquer certaines divergences avec l'approche formalisée des options réelles. Le niveau d'incertitude, qui est théoriquement important dans le recours aux options réelles (Inklaar, Yang, 2012), apparaît n'avoir qu'une influence très marginale sur l'adoption du raisonnement optionnel par les managers. On pourrait avancer que les managers ne sont que faiblement en mesure de donner une estimation fiable du niveau d'incertitude, notion d'ailleurs non mesurable dans sa définition classique (Driouchi, T. David J. Bennett, D. J. 2012). Cependant, il est également possible de supposer que les décideurs adoptent des heuristiques dans le cadre du raisonnement optionnel. Dès lors, le raisonnement par options réelles ne serait pas une simple version allégée ou dégradée du raisonnement optionnel tel qu'il est présenté dans la théorie financière (Adner, Levinthal, 2004). Il y aurait également des éléments supplémentaires, des interférences, ce qui justifie l'étude du « real option reasoning » comme un objet à part entière.

Troisièmement, notre travail présente un intérêt méthodologique. La difficulté d'appréhender concrètement ce qu'est le « real option reasoning » explique probablement que les études cherchant à mesure les options et leurs déterminants de la part des managers soient finalement très rares (Krychowski, 2008). Nous sommes au contraire convaincus qu'il est temps, sur cette question, de tenter d'appréhender concrètement les modes de réflexion des managers sur les projets d'investissement, au besoin en essuyant des imperfections dans l'élaboration des instruments de mesure, qui pourront être affinés par des travaux ultérieurs. 


\section{Limites et voies de recherche}

Notre travail n'est pas, en effet, exempt de critiques. En tant qu'étude exploratoire, il n'aborde qu'une partie de son objet d'étude, à l'aide d'instruments, tous élaborés pour la cause, et qui gagneront probablement à être renforcés. Mais, dans une certaine mesure, ces limites ne sont que la contrepartie d'un champ d'étude extrêmement étendu et qui commence à peine à être exploré. Par ailleurs, On doit remarquer que les modèles 1,2 et 4 présentent des $\mathrm{R}^{2}$ très faibles. Ils n'expliquent donc qu'une très faible part de la variance de la variable dépendante. Ceci suggère que ces modèles doivent être complétés et enrichis par d'autres variables afin de mieux expliquer notre objet de recherche.

Les prolongements de notre travail sont, dans cette perspective, assez manifestes. Une première voie consiste à intégrer la dimension de performance dans les choix. Une fois précisée la mesure et les déterminants du choix optionnel, il convient de s'interroger sur la manière dont la conception d'options réelles peut contribuer à la performance des entreprises. Une deuxième voie est celle d'un rapprochement avec les études de nature cognitive. Si les heuristiques ont un poids qui rend le «real option reasoning» autonome, il convient d'intégrer d'autres variables de modélisation, notamment en termes de rationalité limitée. Cette voie, à peine explorée, recèle des opportunités évidentes.

\section{REFERENCES}

Adner, R., Levinthal, D. A. 2004,Real options and real tradeoffs. Academy of Management Review 29(1): 120-126.

Adner, R., Levinthal, D. A. 2004, Whatis not a real option: Considering boundaries for the application of real options to business strategy. Academy of Management Review 29(1): 7485.

Aguerrevere, F. L. 2003. Equilibrium investment strategies and output price behavior: A realoptions approach. Review of Financial Studies 16(4): 1239-1272.

Alvarez, L. H. R., Keppo, J. 2002. The impact of delivery lags on irreversible investment under uncertainty. European Journal of Operational Research 136(1): 173-180. 
Alvarez, L. H. R., Stenbacka, R. 2003. Optimal risk adoption: A real options approach. Economic Theory 23(1): 123-147.

Bellalah, M. 2001. Irreversibility, sunkcosts and investment under incomplete information. R\&D Management 31(2).

Benaroch, M. 2002. Managing information technology investment risk: A real options perspective. Journal of Management Information Systems 19(2): pp. 43-84.

Bollen N., "Real Options and Product Life Cycles", Management Science, n 45, 1999, p. $670-684$

Bowman, E., H., Moskowitz, G., T., 2001. Real options analysis and strategic decision making. Organization Science 12(6): 772.

Bowman E. H., Hurry D., "Strategy Through the Option Lens: an Integrated View of Ressource Investments and the Incremental-Choice Process", Academy of Management Review, vol. 18, n 4, 1993, p. 760-782.

Bloom, N., Bond, S., Van Reenen, J. 2007. Uncertainty and investment dynamics. Review of EconomicStudies 74(2): 391-415

Bloom, N., Van Reenen, J. 2002. Patents, real options and firm performance. Economic Journal 112(478)

Blum, V., 2012, Options réelles : métamorphoses d'une méthode d'évaluation capturant le risque, Gestion 2000.

Chi, T. 2000. Option to acquire or divest a joint venture. Strategic Management Journal 21(6): 665.

Cassiman, B., Ueda, M. 2006. Optimal project rejection and new firm start-ups. Management Science 52(2): 262-275

Chi T., McGuire D. J., "Collaborative Ventures and Value of Learning: Integrating the Transaction Cost and Strategic Option Perspectives on the Choice of Market Entry Modes", Journal of International Business Studies, second semestre 1996, p. 286-307.

Cohendet P., Llerena P., "Flexibilité et modes d'organisation", Revue française de gestion, $\mathrm{n}^{\circ}$ 123, mars-avril-mai 1999, p. 72-79.

Copeland, T., Antikarov, V., 2001, Real Options : A Practitioner's Guide, Texere Publishing, New York.

Copeland, T., Antikarov, V., 2005, Real Options : Meeting the Georgetown Challenge, Journal of Applied Corporate Finance, vol. 17, $n^{\circ} .2,32-51$. 
Driouchi, T. David J. Bennett, D. J. 2012, Real Options in Management and Organizational Strategy: A Review of Decision-making and Performance Implication, International Journal of Management Reviews, Vol. 14, 39-62.

Durand, R., Gomez, P., Y., Monin, P., 2002, Le management stratégique face à la théorie des options, Revue française de gestion - $\mathrm{N}^{\circ}$ 160/2006, pp 159 - 176.

Folta T., Miller K.D., "Entry Timing and Option Value", Academy of Management Proceedings, 1999.

Goffin, R., 1999, Principes de finance moderne, 2ème édition, Economica.

Henry, C., 1974, Option Values in the Economics of Irreplaceable Assets, Review of Economic Studies, Symposium on the economics of exhaustible resources, p.89-104.

Harchaoui, T. M., Lasserre, P. 2001. Testing the option value theory of irreversible investment. International Economic Review 42(1): 14

Ingersoll, J., Ross, S., 1992, Waiting to invest: investment and uncertainty, Journal of Business, p.1-29.

Inklaar, R., Yang, J., 2012, The impact of financial crises and tolerance for uncertainty. Journal Of Development Economics, vol. 97, $\mathrm{n}^{\circ} 2,466-480$.

Jaeger, M., 1996, Le concept et les modèles d'évaluation d'options, Economies et Société, Vol.22, p.33-60.

Kim, D., J., Kogut. B., 1996, Technological platforms and diversification, Organization Science, Vol.7, p.283- 301.

Kogut B., Kulatilaka N., 1994, Operating Flexibility, Global Manufacturing and the Option Value of a Multinational Network”, Management Science, vol. 40, n 1, p. 123-139.

Kogut,B., 1991, Joint ventures and the option to expand and acquire, Management Science, Vol.37, n 1, p.19 - 33.

Kogut,B., Kulatilaka, N.,1994, Operating flexibility, global manufacturing and the option value of a multinational network», Management Science, Vol.40, n¹, p.123-139.

Kulatilaka, N., Markus, A., J.,1992, Project valuation under uncertainty : When does DCF fail? », Journal of Applied Corporate Finance, Vol.5, n³, p.92-100.

Lakatos, I., 1978, The Methodology of Scientific Research Programme, Philosophical Papersedited by J. Worral et G. Currie, Cambridge University Press, Vol. 1-2.

Krychowski C., 2007, Apport et limites des options réelles à la décision d'investissement stratégique: Une étude appliquée dans le secteur des télécommunications, Thèse HEC Paris. 
Krychowski, C., 2008, Les options réelles sont-elles utiles pour prendre des décisions d'investissement stratégiques ? Une revue de littérature, XVIIème conférence de l'AIMS.

Luehrman, T., 1997, What's it worth ? A general manager's guide to valuation, Harvard Business Review, vol. 75, n 3, 132-142.

Luehrman, T., 1998, Investment opportunities as real options: Getting started on the numbers, Harvard Business Review, vol. 76, $\mathrm{n}^{\circ}$ 4, 51-67.

Majd, S., Pindyck, R., 1987, Time to build, option value and investment decisions, Journal of Financial Economics, Vol.18, p.7-27.

McGrath, R. G., Nerkar, A. 2004. Real options reasoning and a new look at the r\&d investment strategies of pharmaceutical firms. Strategic Management Journal 25(1): 1-22

Mcgrath, R., G., 1999, Falling Forward: Real Options Reasoning And Entrepreneurial Failure, Academy of Management Review, vol. 24, $\mathrm{n}^{\circ}$ 1, 13-30.

Miller, K., et T. Folta, T., 1999, Entry timing and option value, Academy of Management Proceedings.

Myers, S., C., 1977, Determinants of corporate borrowing, Journal of Financial Economics 5(2): 147- 175 .

Myers, S., C., 1996, Fischer black's contributions to corporate finance, Financial Management 25(4): 95-103

Perez, M., H., Berard, C., 2009, Valoriser l'incertitude : Comprendre la dynamique des options réelles, Revue Des Sciences De Gestion, vol. 236, 35-41.

Pindyck, R., 1991, Irreversibility, uncertainty and investment, Journal of EconomicLiterature, Vol. 29, p.1110-1148.

Philippe, H., 2004, Les options réelles: modèle financier ou modèle de gestion? Thèse, ParisDauphine, Paris.

Triantis, A., J., 2005, Realizing the potential of real options: Does theory meet practice? Journal of Applied Corporate Finance 17(2): 8-16

Triantis, A., J., Borison, A., 2001. Real options: State of the practice. Journal of Applied Corporate Finance 14(2): 8-24

Triantis, A. 2005. Realizing the potential of real options: Does theory meet practice? Journal of Applied Corporate Finance 17(2): 8-16

Triantis, A. J., Borison, A. 2001. Real options: State of the practice. Journal of Applied Corporate Finance 14(2): 8-24 
Trigorgis, L, 1996, Real Options: Managerial Flexibility and Strategy in Resource Allocation, Cambridge, The MIT Press.

Trigeorgis, L., 1993, Real options and interactions with financial flexibility, Financial Management, p.202 - 224 .

Verbeeten, F., H., M., 2006, Do organizations adopt sophisticated capital budgeting practices to deal with uncertainty in the investment decision?: A research note. Management Accounting Research 17(1): 106-120.

Weisbrod, B., A., 1964, Collective-consumption services of individual Lacy consumption goods », Quarterly Journal of Economics, Vol.78, n³, p.471-477.

Williams, J., 1991, Real estate development as an option », Journal of Real Estate Finance and Economics, Vol.4, p.191-208.

Ziegler, B., 1996, IBM, Apple, HP to disband Taligent : Big Layoffs Loom at Software Venture », Wall Street Journal. 